It has been accepted for many years that replication of the chromosome in $E$. coli proceeds from a single point sequentially around a continuous genome. The replication origin is generally considered to be independent of the integrated $F^{\prime}$ factor in $H f r$ strains and to be fixed in $F^{-}$strains. A number of independent studies strongly suggest that the replication origin is frequently located in the 6 to 9 o'clock sector of the genetic map of $E$. coli. Helmstetter's approach to this question has been to measure the initial rates of induced enzyme synthesis (tryptophanase, $\beta$-galactosidase, D-serine deaminase) in relation to the chromosome replication cycle ( $J$. Bact., 95, 1634; 1968). The rationale of this approach is based on the substantial claim that the rate of synthesis of an enzyme is related to the number of copies of its structural gene in the cell. The rates of induced synthesis of the three enzymes increased sharply 4, 20 and $34 \mathrm{~min}$, respectively, after the start of a round of replication (the latter defined on the basis of ${ }^{3} \mathrm{H}$-thymidine incorporation to last for $40 \mathrm{~min}$ ). When these data were matched with the ind, lac and $D s d$ loci of the genetic map of strain $K-12$, an estimated position for the replication origin at about 8 o'clock was possible. Helmstetter noted also that new rounds of replication were initiated before the previous round was completed in rapidly growing populations, a phenomenon discovered previously in Bacillus subtitis. Thus, in cells which have overlapping rounds of replication, the sequence of increased enzyme inducibility will differ from the sequence on the genome. For example, since tryptophanase maps 4 min from the origin and the deaminase 7 min from the terminus, the enzyme sequence is altered in cells in which replication rounds overlap by $11 \mathrm{~min}$ or more.

At the present time Helmstetter depicts the coordination between chromosome replication and cell division in $E$. coli $B / r$ as $\cdot$ (1) replication initiated at a fixed point on the genome (8 o'clock); (2) replication time of $40 \mathrm{~min}$ or more; (3) at the end of replication a triggering of cell division (20 min or more). As a result, the rate of cell division is intimately linked to the frequency of initiation.

\section{Following Infections Chemically}

\section{from our Medical Biochemistry Correspondent}

THe usual diagnostic techniques of bacteriology and virology are slow and it would often be useful to have quicker methods of following the progress of an infection so that, when the infecting organisms become resistant to the drugs used, treatment can be changed as quickly as possible. In patients with chronic bronchitis, the DNA content and lactate dehydrogenase (LDH) activity of the sputum have recently been found to be reliable early indications of the presence of infection. (Bürgi, Wiesmann, Richterich, Regli and Mediei, Brit. Med. J., ii, 654; 1968.)

In chronic bronchitic patients, when there was inflammation the DNA concentration of the sputum was high, between 2.9 and $8.7 \mathrm{mg}$ per $\mathrm{ml}$., whereas when there was no evidence of inflammation the DNA concentration varied between 0 and $2.95 \mathrm{mg}$ per $\mathrm{ml}$. There was also a significant difference in $\mathbf{L D H}$ activity between the two groups, but there was also more overlap between the groups. When Bürgi et al. investigated the isozymes of IDDH by electrophoresis they found there was a great difference between the two groups. In the sputum from inflammatory secretions the isozymes were mainly types 4 and 5 , suggesting that the activity came from leucocytes or possibly mucosal cells. When there was no inflammation there was often no LDH activity in the sputum, but if there was activity it was mainly isozymes 1-3, which are those found in normal serum. When the infections were treated with antibiotics, both DNA and LDH in the sputum fell as the infection responded. LDH seemed to be the more sensitive indicator, as the measured activity fell the day before a fall in DNA concentration could be detected. On relapse the $\mathrm{LDH}$ activity also proved to be the most sensitive indicator of the emergence of drug-resistant bacteria. The LDH activity rose before the DNA concentration and several days before any clinical signs of a return of infection.

The work of Mitruka, Alexander and Carmichel (Science, 160, 309; 1968) on some viruses infecting dogs suggests that it may soon be possible to diagnose as well as to follow the progress of infections by detection of metabolites in the serum. They examined by gas chromatography the volatile components of the serum in 11 dogs before and after infection with canine infectious hepatitis virus. In 10 out of 11 dogs a now peak with a retention time of 420 seconds was present in the first sample taken after infection (the peak appeared in the second sample in the eleventh dog). This peak was present in all the serum samples after infection in the dogs which dicd, but began to disappear about the seventh day in the dogs which recovered. The dogs which died were also distinguished by the appearance of a second peak with a retention time of 1,380 seconds, which was never seen in any sample from the animals which recovered.

To compare the effects of different viruses, they inoculated dog kidney cells in tissue culture with the hepatitis virus, strain $F-205$ of the canine herpes virus, the Rockborn strain of the canine distemper virus and a parainfluenza virus (resembling simian virus 5). They found nine new components in infected cultures which were not present in non-infected cultures but which did not appear to be specific. More interesting was the discovery that each virus gave rise to some new components which were unique to that virus. Cultures containing the hepatitis virus gave the same compounds with retention times of 420 and 1,380 seconds which were found in the whole animal experiments. The herpes virus gave 4 new peaks, the distcmper virus 3 and the parainfluenza virus 2 , which seemed to be unique to the particular virus. Obviously much more needs to be known about how specific these compounds are as indicators of infection, but, if they are unique, gas chromatography of serum metabolites could give very rapid diagnoses. Fstimation of the quantities of metabolites present in the serum might also be a useful means of following the progress of infections and the results of therapy.

\section{Biological Macromolecules}

\section{from a Correspondent}

A symposium entitled "Interactions between Subunits of Biological Macromolecules" and sponsored by the Commission on Molecular Biophysics of the International Union for Pure and Applied Biophysics was held 